\title{
捕获分支是拟圆: 具有临界不动点的三次多项式
}

献给杨乐教授 80 华诞

\section{王悦洋, 邱维元*}

复旦大学数学科学学院, 上海 200433

E-mail: yueyangwang@icloud.com,wyqiu@fudan.edu.cn

收稿日期: 2018-09-25; 接受日期: 2019-02-19; 网络出版日期: 2019-05-17; * 通信作者

国家自然科学基金 (批准号: 11671091 和 11731003) 资助项目

摘要 按 Milnor 分类, 具有有限临界不动点的三次多项式的参数平面中, 有界双曲分支可以分为三 类: 主双曲分支、捕获分支、非捕获分支. Roesch 证明了主双曲分支边界具有稠密尖点, 本文证明捕 获分支的边界是拟圆周, 从而没有尖点. 同时, 非捕获分支的边界是一条解析曲线 (至多有一个例外的 尖点).

关键词 三次多项式双曲分支捕获分支拟圆周 尖点 全纯运动

MSC (2010) 主题分类 $37 \mathrm{~F} 45,37 \mathrm{~F} 10$

\section{1 引言}

Branner 和 Hubbard ${ }^{[1]}$ 首先研究了复三次多项式 $z \mapsto z^{3}+a z+b(a, b \in \mathbb{C})$ 的参数空间 $\mathcal{S}$. 他们 证明了其连通轨迹 (具有连通 Julia 集的参数集) 是 $\mathcal{S}$ 中的单连通紧集. Petersen 和 Tan ${ }^{[2]}$ 对 $\mathcal{S}$ 中主 双曲分支的拓扑进行了刻画. 然而, 由于 $\mathcal{S}$ 的参数空间是复 2 维 (实 4 维) 的, 其连通轨迹和双曲分支 的边界通常是复杂的分形, 很难给出一个比较清晰的直观描述, 因此, Milnor ${ }^{[3]}$ 建议研究 $\mathcal{S}$ 中的复一 维截面 $\mathcal{S}_{p}$, 其中 $\mathcal{S}_{p}$ 是具有周期为 $p$ 的周期临界点的三次多项式全体. 其中最简单的是 $\mathcal{S}_{1}$, 即有一个 有限临界不动点的三次多项式全体. $\mathcal{S}_{1}$ 中的三次多项式都可以写成如下形式:

$$
f_{a}(z)=z^{3}-3 a^{2} z+2 a^{3}+a, \quad a \in \mathbb{C},
$$

其中参数 $a$ 即为 $f_{a}$ 的临界不动点. 因此, 我们可以将 $\mathcal{S}_{1}$ 用复平面 $\mathbb{C}$ 参数化. 以后, 我们将 $\mathcal{S}_{1}$ 等同 于其参数平面 $\mathbb{C}$.

Faught ${ }^{[4]}$ 讨论了 $\mathcal{S}_{1}$ 的连通轨迹和双曲分支的拓扑性质. Roesch ${ }^{[5]}$ 对此进行了更细致和更为严 格的研究, 并将其推广到一类较广的函数族: 具有最高阶临界不动点的多项式函数族, 即临界不动点 
的阶等于多项式次数减 $1, \mathcal{S}_{1}$ 是临界不动点阶为 2 的特殊情形. 已知 $\mathcal{S}_{1}$ 中的连通轨迹 $\mathcal{C}_{1}$ 是参数平 面 $\mathbb{C}$ 中的单连通紧集, 其余集是唯一的无界双曲分支, 而包含在连通轨迹 $\mathcal{C}_{1}$ 中的双曲分支都是有界 单连通区域. Faught ${ }^{[4]}$ 和 Roesch ${ }^{[5]}$ 进一步证明了包含在连通轨迹 $\mathcal{C}_{1}$ 内的双曲分支的边界是 Jordan 曲线, 并且, 如果二次多项式的 Mandelbrot 集是局部连通的, 则 $\mathcal{S}_{1}$ 的连通轨迹 $\mathcal{C}_{1}$ 也是局部连通的.

上述已有性质均是拓扑性质, 本文将关注的是包含在 $\mathcal{S}_{1}$ 的连通轨迹中的有界双曲分支边界的几 何性质. 注意到, $\mathcal{S}_{1}$ 中的每个多项式 $f_{a} \in \mathcal{S}_{1}$ 有两个临界点 $\pm a$, 其中 $a$ 是超吸引不动点, 而 $-a$ 的轨 道有各种不同的状态, 称为自由临界点. 按照 Milnor ${ }^{[3]}$ 的分类, 连通轨迹 $\mathcal{C}_{1}$ 中的双曲分支可以分为 以下三类:

(1) 主双曲分支: 自由临界点 $-a$ 位于超吸引不动点 $a$ 的直接吸引域内;

(2) 捕获分支: 自由临界点 $-a$ 位于超吸引不动点 $a$ 的吸引域内, 但不在 $a$ 的直接吸引域内;

(3) 非捕获分支: 自由临界点 $-a$ 不在超吸引不动点 $a$ 的吸引域内.

主双曲分支只有一个, 即包含参数 $a=0$ 的双曲分支, 记主双曲分支为 $\mathcal{H}_{0}$. Roesch ${ }^{[5]}$ 详细研究了 $\mathcal{H}_{0}$ 的边界, 证明在 $\partial \mathcal{H}_{0}$ 上有可数多个抛物参数, 这些抛物参数在 $\partial \mathcal{H}_{0}$ 上是稠密的, 并且每个抛物参 数同时位于某个 Mandelbrot 集拟共形同胚象 (小 Mandelbrot 集) 的尖点处. 从而, $\mathcal{H}_{0}$ 的边界是一条 具有稠密尖点的 Jordan 曲线.

一个自然的问题是, 除了主双曲分支, 捕获分支和非捕获分支的边界是怎样的一条曲线, 是否有 尖点? 本文将回答这个问题, 证明: 捕获分支的边界没有尖点, 它是一个拟圆周. 而非捕获分支的边界 是光滑曲线, 至多有一个例外的尖点.

定理 1.1 在 $\mathcal{S}_{1}$ 的参数平面中, 每个捕获分支的边界都是拟圆周.

定理 1.2 在 $\mathcal{S}_{1}$ 的参数平面中, 非捕获分支的边界是一条除了在乘子为 1 的抛物参数处可能有 一个尖点外的解析曲线.

定理 1.2 的证明类似于 Mandelbrot 集内双曲分支. 事实上，非捕获分支内的多项式是可重整的， 每个非捕获分支包含在某个小 Mandelbrot 集内. 本文主要工作是证明定理 1.1, 证明将在第 3 节中 给出.

图 1 显示了 $\mathcal{S}_{1}$ 中连通轨迹 $\mathcal{C}_{1} 、 \mathcal{C}_{1}$ 中主双曲分支 $\mathcal{H}_{0}($ 图 1(a))、捕获分支 $\mathcal{V}($ 图 1(b)) 和非捕获 分支 $\mathcal{N}($ 图 1(c)). 图中所显示的捕获分支的边界都是拟圆周.

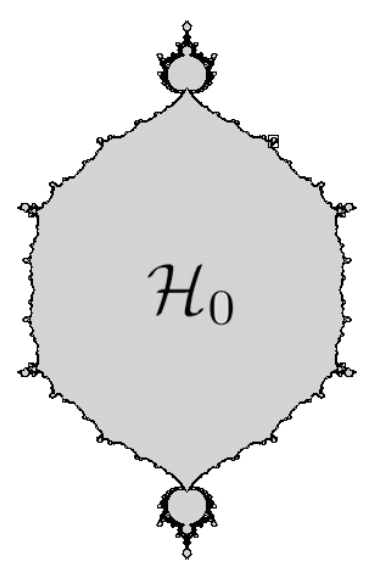

(a)

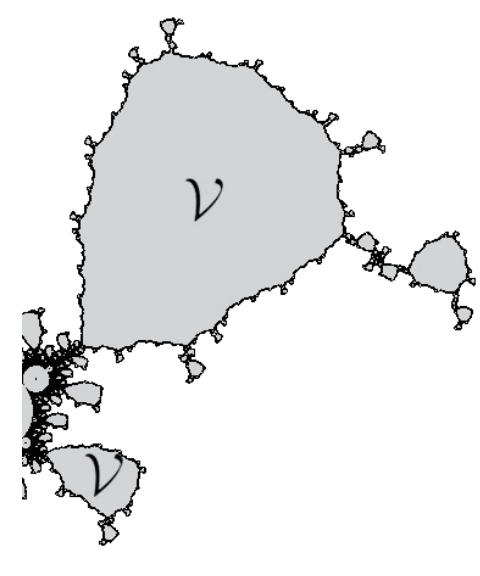

(b)

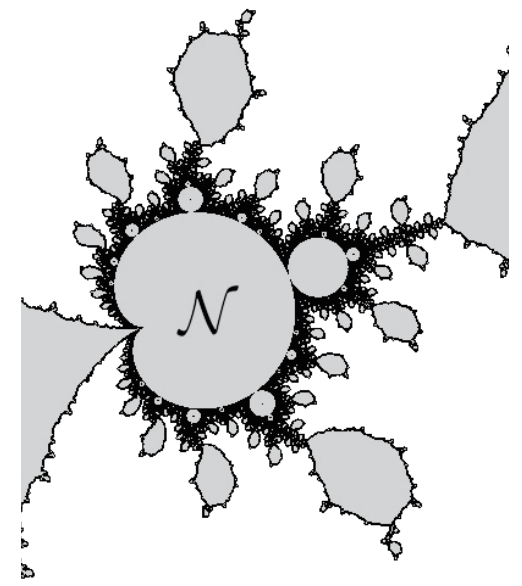

(c)

图 $1 \mathcal{S}_{1}$ 中连通轨迹 $\mathcal{C}_{1}$, 主双曲分支 $\mathcal{H}_{0}(\mathrm{a})$ 和捕获分支 $\mathcal{V}(\mathrm{b})$ 以及非捕获分支 $\mathcal{N}(\mathrm{c})$ 


\section{2 定义和准备知识}

首先介绍有关定义和准备知识, 细节可参见文献 [3-5]. 已知 $\mathcal{S}_{1}$ 中多项式可参数化表示为

$$
f_{a}(z)=z^{3}-3 a^{2} z+2 a^{3}+a, \quad a \in \mathbb{C} .
$$

$f_{a}$ 有两个有限临界点 $\pm a$, 其中 $a$ 是 $f_{a}$ 的超吸引不动点, $-a$ 有不同的轨道状态, 称为自由临界点. 记 $f_{a}^{m}$ 是 $f_{a}$ 的 $m$ 次迭代, 定义

$$
\mathcal{H}=\left\{a \in \mathbb{C}: \lim _{m \rightarrow \infty} f_{a}^{m}(-a)=a\right\},
$$

那么, $\mathcal{H} \subset \mathcal{C}_{1}$ 是开集, $\mathcal{H}$ 的每个连通分支是 $\mathcal{S}_{1}$ 的双曲分支. 称 $\mathcal{H}$ 中包含 0 的连通分支为主双曲分支, 记为 $\mathcal{H}_{0} . \mathcal{H} \backslash \mathcal{H}_{0}$ 中的每个连通分支称为捕获分支. $\mathcal{C}_{1} \backslash \mathcal{H}$ 中的每个双曲分支称为非捕获分支.

记 $a$ 的直接吸引域为 $U_{a}$, 则 $a \in \mathcal{H}_{0}$ 当且仅当 $-a \in U_{a} ; a \in \mathcal{H} \backslash \mathcal{H}_{0}$ 当且仅当 $-a \notin U_{a}$ 但存在正 整数 $m \geqslant 1$ 使得 $f_{a}^{m}(-a) \in U_{a}$. 如果 $a$ 属于某个非捕获分支, 则对任意 $m \geqslant 0$, 都有

$$
f_{a}^{m}(-a) \notin U_{a}
$$

已知 $U_{a}$ 是有界单连通区域, Faught ${ }^{[4]}$ 进一步证明下面的引理:

引理 2.1 对任意 $a \in \mathbb{C}, \partial U_{a}$ 是 Jordan 曲线.

如果 $a \in \mathcal{H}$, 那么, $f_{a}$ 是双曲的, 由文献 [6] 的结论知下面的引理:

引理 2.2 如果 $a \in \mathcal{H}$, 则 $\partial U_{a}$ 是拟圆周.

注 2.1 引理 2.1 对任意次数大于等于 2 的多项式的吸引或抛物 Fatou 分支都成立, 引理 2.2 对 临界非回归多项式的吸引或抛物 Fatou 分支 (抛物分支时, 乘子为 1 的分支除外) 成立, 参见文献 [7].

在超吸引不动点 $a$ 的邻域内, 有 Böttcher 坐标 $\beta_{a}(z)$ 满足

$$
\beta_{a} \circ f_{a}(z)=\left(\beta_{a}(z)\right)^{2}, \quad \beta_{a}(a)=0 .
$$

如果 $a \in \mathcal{H}_{0}$, 那么, $\beta_{a}$ 可以扩充到包含 $a$ 的某个闭拓扑圆, 使得 $-a$ 在其边界上, 且 $\beta_{a}$ 在其上是同胚, 在其内部是共形的. 这样, $\beta_{a}(-a)$ 是良定义的. 记 $\eta(a)=\beta_{a}(-a), a \in \mathcal{H}_{0}$, 记 $\mathbb{D}$ 为单位圆, 则有如下引 理 (证明参见文献 [3]).

引理 2.3 映射 $\eta: \mathcal{H}_{0} \rightarrow \mathbb{D}, \eta(a)=\beta_{a}(-a)$, 是一个两层全纯分支覆盖, 其唯一的分支点为 0 .

由此, $\mathcal{H}_{0}$ 是单连通的, 并可在 $\mathcal{H}_{0}$ 上引入参数内射线: 对 $t \in \mathbb{R} / \mathbb{Z}$, 以角度为 $t$ 的参数内射线定 义为

$$
\mathcal{R}_{\mathcal{H}_{0}}^{t}=\left\{\eta^{-1}\left(r \mathrm{e}^{\mathrm{i} 2 \pi t}\right): r \in[0,1)\right\} .
$$

如果 $a \in \mathcal{H} \backslash \mathcal{H}_{0}$, 则临界点 $-a \notin U_{a}$. Böttcher 坐标 $\beta_{a}$ 可以扩充到整个 $U_{a}$ 上, 使得 $\beta_{a}: U_{a} \rightarrow \mathbb{D}$ 为共形映射. 由引理 $2.1, \beta_{a}$ 可以扩充为 $\bar{U}_{a}$ 到 $\overline{\mathbb{D}}$ 的同胚, 且仍满足

$$
\beta_{a} \circ f_{a}(z)=\left(\beta_{a}(z)\right)^{2}
$$

设 $\mathcal{V} \subset \mathcal{H} \backslash \mathcal{H}_{0}$ 是一个捕获分支. 按定义, 对每个 $a \in \mathcal{V}$, 存在一个最小的正整数 $m=m(a)$ 使得 $f_{a}^{m}(-a) \in U_{a}$, 即

$$
m(a)=\min \left\{m \in \mathbb{N}: f_{a}^{m}(-a) \in U_{a}\right\}, \quad a \in \mathcal{V}
$$


利用当 $a$ 在双曲分支内时, $\partial U_{a}$ 连续依赖于参数 $a$ (在 Hausdorff 拓扑下, 参见文献 [8]), 容易得到, 如 果对某个 $a_{0} \in \mathcal{V}$, 有 $f_{a_{0}}^{m}\left(-a_{0}\right) \in U_{a_{0}}$, 但 $f_{a_{0}}^{m-1}\left(-a_{0}\right) \notin U_{a_{0}}$, 那么, 存在 $a_{0}$ 的某个邻域 $\mathcal{N}_{\varepsilon}$, 使得当 $a \in \mathcal{N}_{\varepsilon}$ 时, 仍然有

$$
f_{a}^{m}(-a) \in U_{a}, \quad f_{a}^{m-1}(-a) \notin U_{a} .
$$

因此, $m=m(a)$ 局部是常数. 由 $\mathcal{V}$ 的连通性, 有下面的引理:

引理 $2.4 m=m(a)$ 在 $\mathcal{V}$ 上是常数.

称上述 $m \in \mathbb{N}$ 为捕获分支 $\mathcal{V}$ 的阶. 下面的引理给出了 $\mathcal{V}$ 上的 Riemann 映射 (证明参见文献 [5, 引理 2.8]).

引理 2.5 设 $\mathcal{V}$ 是 $m$ 阶捕获分支, 则

$$
\phi: \mathcal{V} \rightarrow \mathbb{D}, \quad \phi(a)=\beta_{a} \circ f_{a}^{m}(-a)
$$

是一个共形映射.

注 2.2 在 Roesch 的论文中, $f_{a}$ 的参数化稍有不同, 她将临界不动点规范化为 0 而不是 $a$, 下同.

推论 2.1 对给定 $m \in \mathbb{N}$, 仅有有限多个 $m$ 阶捕获分支.

证明 由引理 2.5 知, $\phi: \mathcal{V} \rightarrow \mathbb{D}$ 是共形映射, 因此, $a=\phi^{-1}(0) \in \mathcal{V}$, 且 $\beta_{a} \circ f_{a}^{m}(-a)=\phi(a)=0$. 又当 $a$ 在捕获分支 $\mathcal{V}$ 内时, Böttcher 映射 $\beta_{a}: U_{a} \rightarrow \mathbb{D}$ 也是共形映射, 且 $\beta_{a}(a)=0$, 因此, $f_{a}^{m}(-a)=a$, 即 $a=\phi^{-1}(0) \in \mathcal{V}$ 是这个代数方程的一个根. 从而捕获分支的个数不超过方程 $f_{a}^{m}(-a)=a$ 根的个数, 因而是有限的.

下面的结论是我们要用到的关键结论之一 (参见文献 [5, 引理 1]).

引理 2.6 $\mathcal{H}$ 中任意两个双曲分支的闭包是互不相交的.

定理 $2.1^{[4,5]} \mathcal{H}$ 中的每个双曲分支的边界都是 Jordan 曲线.

上面两个结论表明, 主双曲分支 $\mathcal{H}_{0}$ 和任意捕获分支 $\mathcal{V}$ 都是 Jordan 区域, 且 $\overline{\mathcal{H}}_{0} \cap \overline{\mathcal{V}}=\emptyset$. 对任意 两个互不相同的捕获分支 $\mathcal{V}_{1}$ 和 $\mathcal{V}_{2}$, 有 $\overline{\mathcal{V}}_{1} \cap \overline{\mathcal{V}}_{2}=\emptyset$.

\section{3 定理的证明}

定理 1.1 的证明的主要工具是全纯运动和 $\lambda$ - 引理. 设 $\mathcal{W}$ 是单连通区域, $A \subset \mathbb{C}$ 是复平面上子集, $w_{0} \in \mathcal{W}$. 映射 $h: \mathcal{W} \times A \rightarrow \mathbb{C}$ 满足如下条件:

(1) $h_{w_{0}}(z):=h\left(w_{0}, z\right)$ 是恒等映射;

(2) 对给定 $w \in \mathcal{W}, h_{w}(z):=h(w, z)$ 是 $A$ 上单射;

(3) 对给定 $z \in A$, 映射 $w \mapsto h(w, z)$ 是 $\mathcal{W}$ 上全纯函数,

那么, 称 $h$ 是 $\mathcal{W} \times A$ 上以 $w_{0}$ 为基点的全纯运动.

定理 $3.1\left(\lambda\right.$-引理 $\left.{ }^{[8-10]}\right)$ 设 $\mathcal{W}$ 是单连通双曲区域, $A \subset \mathbb{C}, w_{0} \in \mathcal{W} . h: \mathcal{W} \times A \rightarrow \mathbb{C}$ 是以 $w_{0}$ 为 基点的全纯运动, 那么, $h$ 可以扩充为 $\mathcal{W} \times \mathbb{C}$ 上全纯运动 $\bar{h}: \mathcal{W} \times \mathbb{C} \rightarrow \mathbb{C}$. 进一步, 对给定 $w \in \mathcal{W}$, 映 射 $h_{w}: \mathbb{C} \rightarrow \mathbb{C}, h_{w}(z)=h(w, z)$, 是一个拟共形同胚, 其 Beltrami 系数 $\mu_{w}(z)=\bar{\partial} h_{w}(z) / \partial h_{w}(z)$ 满足

$$
\left\|\mu_{w}\right\|_{\infty}=\operatorname{ess~sup}_{z \in \mathbb{C}}\left|\mu_{w}(z)\right| \leqslant \frac{\mathrm{e}^{\rho\left(w, w_{0}\right)}-1}{\mathrm{e}^{\rho\left(w, w_{0}\right)}+1}<1,
$$

其中 $\rho\left(w, w_{0}\right)$ 是 $\mathcal{W}$ 上 $w$ 和 $w_{0}$ 之间的双曲距离. 
引理 3.1 设 $\mathcal{W} \subset \mathbb{C}$ 是单连通区域, 满足 $\overline{\mathcal{W}} \cap \overline{\mathcal{H}}_{0}=\emptyset$. 给定 $a_{0} \in \mathcal{W}$. 那么存在 $\mathcal{W} \times \bar{U}_{a_{0}}$ 上以 $a_{0}$ 为基点的全纯运动 $h: \mathcal{W} \times \bar{U}_{a_{0}} \rightarrow \mathbb{C}$.

证明 由于 $\mathcal{W} \cap \overline{\mathcal{H}}_{0}=\emptyset$, 对任意 $a \in \mathcal{W}, U_{a}$ 上的 Böttcher 坐标 $\beta_{a}$ 可以扩充为 $\bar{U}_{a}$ 到 $\overline{\mathbb{D}}$ 的同 胚. 令

$$
h_{a}(z)=\beta_{a}^{-1} \circ \beta_{a_{0}}(z), \quad z \in \bar{U}_{a_{0}},
$$

则 $h_{a}(z)$ 是良定义的, 且 $h_{a}: \bar{U}_{a_{0}} \rightarrow \bar{U}_{a}$ 是同胚, 因而是单射. 又已知 $\beta_{a}$ 全纯依赖于参数 $a$, 因而, $h_{a}(z)$ 关于参数 $a \in \mathcal{W}$ 全纯. 显然, $h_{a_{0}}(z) \equiv z$ 是恒等映射. 因此, $h: \mathcal{W} \times \bar{U}_{a_{0}} \rightarrow \mathbb{C}, h(a, z)=h_{a}(z)$, 是 全纯运动.

现设 $\mathcal{V}$ 是一个阶为 $m$ 的捕获分支.

引理 3.2 存在 Jordan 区域 $\mathcal{U}$ 和 $\mathcal{W}$ 满足 $\overline{\mathcal{V}} \subset \mathcal{U}, \overline{\mathcal{U}} \subset \mathcal{W}$, 使得

$$
\overline{\mathcal{W}} \cap \overline{\mathcal{H}}_{0}=\emptyset,
$$

且对任意阶不超过 $m$ 的捕获分支 $\mathcal{V}^{\prime}$, 若 $\mathcal{V}^{\prime} \neq \mathcal{V}$, 则有 $\overline{\mathcal{W}} \cap \overline{\mathcal{V}^{\prime}}=\emptyset$. 进一步, 对 $a_{0}=\phi^{-1}(0) \in \mathcal{V}$, 由引 理 3.1 定义的全纯运动 $h: \mathcal{W} \times U_{a_{0}} \rightarrow \mathbb{C}$ 可以扩充为 $\mathcal{W} \times \mathbb{C}$ 上的全纯运动, 且存在 $0<k<1$ 使得对 任意 $a \in \overline{\mathcal{U}}, h_{a}(z)=h(a, z)$ 的 Beltrami 系数满足

$$
\left\|\mu_{a}\right\|_{\infty} \leqslant k<1 .
$$

证明 由定理 2.1 知, $\overline{\mathcal{V}}$ 是闭 Jordan 区域. 再由引理 2.6, 可取到 Jordan 区域 $\mathcal{W}$ 使得 $\overline{\mathcal{V}} \subset \mathcal{W}$, 且 $\overline{\mathcal{W}} \cap \overline{\mathcal{H}}_{0}=\emptyset$. 又由推论 2.1 知, 阶不超过 $m$ 的捕获分支只有有限多个, 故结合引理 2.6 , 适当缩小 $\mathcal{W}$, 可使得对任意阶不超过 $m$ 且不为 $\mathcal{V}$ 本身的捕获分支 $\mathcal{V}^{\prime}$ 都有 $\overline{\mathcal{W}} \cap \overline{\mathcal{V}^{\prime}}=\emptyset$. 进一步取 Jordan 区域 $\mathcal{U}$, 使得 $\overline{\mathcal{V}} \subset \mathcal{U}, \overline{\mathcal{U}} \subset \mathcal{W}$.

现设 $h: \mathcal{W} \times U_{a_{0}} \rightarrow \mathbb{C}$ 是引理 3.1 中定义的全纯运动, 根据定理 $3.1, h$ 可以扩充为 $\mathcal{W} \times \mathbb{C}$ 上的 全纯运动, 使得 $h_{a}(z)=h(a, z)$ 是 $\mathbb{C}$ 上拟共形映射. 由于 $\overline{\mathcal{U}} \subset \mathcal{W}$, 则存在 $R>0$ 使得对任意 $a \in \overline{\mathcal{U}}$, 有 $0 \leqslant \rho\left(a, a_{0}\right) \leqslant R$, 其中 $\rho\left(a, a_{0}\right)$ 是 $\mathcal{W}$ 上的双曲距离. 由 $(3.1)$, 取 $k=\left(\mathrm{e}^{R}-1\right) /\left(\mathrm{e}^{R}+1\right)$, 则对任意 $a \in \overline{\mathcal{U}}, h_{a}$ 的 Beltrami 系数 $\mu_{a}(z)$ 满足 (3.3).

现在, 我们通过 $h_{a}$ 建立参数平面到 $f_{a_{0}}$ 的动力学平面之间的映射. 定义映射

$$
H: \mathcal{W} \rightarrow \mathbb{C}, \quad H(a)=h_{a}^{-1} \circ f_{a}^{m}(-a) .
$$

注意到 $h_{a}$ 已经扩充为 $\mathbb{C}$ 上拟共形映射, 上述映射 $H$ 是良定义的.

引理 3.3 映射 $H$ 是 $\mathcal{U}$ 上拟正则映射.

证明 只需证明存在常数 $k \in[0,1)$ 使得

$$
\left\|\frac{\partial H / \partial \bar{a}}{\partial H / \partial a}\right\|_{\infty}=\underset{a \in \mathcal{U}}{\operatorname{ess} \sup }\left|\frac{\partial H / \partial \bar{a}}{\partial H / \partial a}\right| \leqslant k<1
$$

其中 $\partial H / \partial a$ 和 $\partial H / \partial \bar{a}$ 是局部 $L^{2}$ 可积的广义导数.

为此, 考虑方程 $h_{a} \circ H(a)=f_{a}^{m}(-a)$ 关于 $a$ 和 $\bar{a}$ 的广义偏导数. 由于 $h_{a}$ 和 $f_{a}^{m}(-a)$ 都是关于 $a$ 的全纯函数, 故

$$
\frac{\partial h_{a}}{\partial \bar{a}}=\frac{\partial f_{a}^{m}(-a)}{\partial \bar{a}}=0,
$$


我们得到

$$
\left.\left.\frac{\partial h_{a}}{\partial z}\right|_{H(a)} \frac{\partial H}{\partial \bar{a}}\right|_{a}+\left.\left.\frac{\partial h_{a}}{\partial \bar{z}}\right|_{H(a)} \frac{\partial \bar{H}}{\partial \bar{a}}\right|_{a}=0
$$

于是,

$$
\left.\left.\left|\frac{\partial H / \partial \bar{a}}{\partial H / \partial a}\right|_{a}|=| \frac{\partial H / \partial \bar{a}}{\partial \bar{H} / \partial \bar{a}}\right|_{a}|=| \frac{\partial h_{a} / \partial \bar{z}}{\partial h_{a} / \partial z}\right|_{H(a)}|=| \mu_{a}(H(a)) \mid .
$$

由引理 3.2 知, 存在 $0 \leqslant k<1$ 使得对任意 $a \in \overline{\mathcal{U}}$, 有

$$
\left\|\mu_{a}\right\|_{\infty}=\underset{z \in \mathbb{C}}{\operatorname{ess} \sup _{0}}\left|\mu_{a}(z)\right| \leqslant k
$$

因此,

$$
\left\|\frac{\partial H / \partial \bar{a}}{\partial H / \partial a}\right\|_{\infty}=\underset{a \in \mathcal{U}}{\operatorname{ess} \sup }\left|\frac{\partial H / \partial \bar{a}}{\partial H / \partial a}\right|=\underset{a \in \mathcal{U}}{\operatorname{ess} \sup }\left|\mu_{a}(H(a))\right| \leqslant k<1 .
$$

设 $\mu^{*}=(\partial H / \partial \bar{a}) /(\partial H / \partial a)$. 由 Ahlfors-Bers 可测 Riemann 映射定理 [11] 可知, 存在拟共形映射 $g: \mathcal{U} \rightarrow g(\mathcal{U})$ 满足 $\partial g / \partial \bar{a}=\mu^{*} \partial g / \partial a$. 那么, $H_{1}=H \circ g^{-1}$ 是全纯映射, 因此, $H=H_{1} \circ g$ 是拟正则 映射.

定理 1.1 的证明 (1) 由定义 (3.4) 以及 (2.2) 和 (3.2) 知, 当 $a \in \mathcal{V}$ 时,

$$
H(a)=h_{a}^{-1} \circ f_{a}^{m}(-a)=\beta_{a_{0}}^{-1} \circ \beta_{a} \circ f_{a}^{m}(-a)=\beta_{a_{0}}^{-1} \circ \phi(a) .
$$

已知 Böttcher 坐标 $\beta_{a}$ 是 $U_{a}$ 到 $\mathbb{D}$ 的共形映射, 而由引理 2.5 知, $\phi$ 是 $\mathcal{V}$ 到 $\mathbb{D}$ 的共形映射, 因此, 限制 在 $\mathcal{V}$ 上, $H$ 是 $\mathcal{V}$ 到 $U_{a_{0}}$ 的共形映射. 又由于 $\partial \mathcal{V}$ 和 $\partial U_{a_{0}}$ 都是 Jordan 曲线 (见引理 2.1 和定理 2.1), 因此, 由共形映射的边界对应可得 $H$ 是 $\overline{\mathcal{V}}$ 到 $\bar{U}_{a_{0}}$ 的同胚, $H(\partial \mathcal{V})=\partial U_{a_{0}}$.

(2) 我们证明 $H^{-1}\left(\bar{U}_{a_{0}}\right)=\overline{\mathcal{V}}$, 即如果 $a \in \mathcal{W}$, 且 $H(a) \in \bar{U}_{a_{0}}$, 则必有 $a \in \overline{\mathcal{V}}$. 先证当 $H(a) \in U_{a_{0}}$ 时, $a \in \mathcal{V}$. 由于 $H(a)=h_{a}^{-1} \circ f_{a}^{m}(-a)$, 而 $h_{a}$ 是 $\mathbb{C} \rightarrow \mathbb{C}$ 的同胚, 且 $h_{a}\left(U_{a}\right)=U_{a_{0}}$, 可知 $f_{a}^{m}(-a) \in U_{a}$. 若 $a \notin \mathcal{V}$, 存在最小的整数 $n$ 满足 $0 \leqslant n \leqslant m$ 使得 $f_{a}^{n}(-a) \in U_{a}$, 即 $a \in \mathcal{H}_{0}(n=0)$ 或 $a$ 属于某个阶 为 $n$ 的捕获分支 $\mathcal{V}^{\prime}, \mathcal{V}^{\prime} \neq \mathcal{V}$. 但由引理 3.2 , 有

$$
\mathcal{H}_{0} \cap \mathcal{W}=\emptyset, \quad \mathcal{V}^{\prime} \cap \mathcal{W}=\emptyset,
$$

矛盾. 因此, $a \in \mathcal{V}$ 得证. 现设 $H(a) \in \partial U_{a_{0}}$. 由于 $H$ 是拟正则的 (见引理 3.3), 且不为常值, 故 $H$ 是 一个开映射, $H$ 将 $a$ 的邻域映成 $H(a) \in \partial U_{a_{0}}$ 的某个邻域. 因此, 对 $a$ 的任意充分小邻域 $N(a)$, 存在 $b, b^{\prime} \in N(a)$ 使得 $H(b) \in U_{a_{0}}, H\left(b^{\prime}\right) \notin U_{a_{0}}$. 由前面的讨论即得 $b \in \mathcal{V}, b^{\prime} \notin \mathcal{V}$. 因此, $a \in \partial \mathcal{V}$.

(3) 我们证明: 存在 $\overline{\mathcal{V}}$ 的某个邻域 $\mathcal{U}^{\prime}$, 使得 $H$ 限制在 $\mathcal{U}^{\prime}$ 上是拟共形映射. 令 $\mathcal{U}$ 是引理 3.2 中所 取区域, 满足 $\overline{\mathcal{V}} \subset \mathcal{U}, \overline{\mathcal{U}} \subset \mathcal{W}$. 记 $\gamma=\partial \mathcal{U}$, 则 $\gamma$ 是一条 Jordan 曲线, $\gamma \cap \overline{\mathcal{V}}=\emptyset$. 令 $\Gamma=H(\gamma)$, 则由 (2), 可得

$$
\Gamma \cap \bar{U}_{a_{0}}=\emptyset .
$$

记 $U^{\prime}$ 是 $\mathbb{C} \backslash \Gamma$ 包含 $\bar{U}_{a_{0}}$ 的连通分支. 由于 $H$ 是拟正则映射, 为一个全纯映射和一个拟共形映射的复 合, 因此, 辐角原理仍然成立. 由第一部分讨论, 对 $w \in U_{a_{0}}$, 有唯一的值 $a \in \mathcal{U}$ 使得 $a=H^{-1}(w)$. 由 辐角原理, 这意味着 $\Gamma$ 关于 $w$ 的绕数等于 1 . 从而, 对任意 $w \in U^{\prime}, \Gamma$ 关于 $w$ 的绕数等于 1 . 因此, 再 由辐角原理, $H^{-1}(w)$ 在 $\mathcal{U}$ 有唯一的值. 令 $\mathcal{U}^{\prime}$ 是 $H^{-1}\left(U^{\prime}\right)$ 包含 $\overline{\mathcal{V}}$ 的连通分支, 则 $H: \mathcal{U}^{\prime} \rightarrow U^{\prime}$ 是同 胚, 从而是拟共形映射.

由于已知 $\partial U_{a_{0}}$ 是拟圆周 (引理 2.2), 因此, $\partial \mathcal{V}=H^{-1}\left(\partial U_{a_{0}}\right)$ 是拟圆周. 
定理 1.2 的证明 定理的证明类似于二次多项式 Mandelbrot 集内的双曲分支 (参见文献 [12]). 设 $\mathcal{N}$ 是一个非捕获分支, 那么, 对任意 $a \in \mathcal{N}, f_{a}$ 的临界点 $-a$ 不属于临界不动点 $a$ 的吸引域. 因此, 必定被一个不同于临界不动点 $a$ 的吸引周期轨道所吸引, 设这个吸引周期轨道为

$$
\mathcal{O}(a)=\left\{z_{0}(a), z_{1}(a), \ldots, z_{p-1}(a)\right\}
$$

周期为 $p=p(a)$, 乘子为 $\lambda(a)=\left(f_{a}^{p}\right)^{\prime}\left(z_{0}(a)\right)$, 满足 $|\lambda(a)|<1$. 由于除 $a$ 外, $f_{a}$ 只有唯一的临界点 $-a$, 而每个吸引周期轨道至少吸引一个临界点, 因此, 吸引周期轨道 $\mathcal{O}(a)$ 是唯一的. 由隐函数定理知, 每 个周期点 $z_{j}(a) \in \mathcal{O}(a)$ 可以延拓为 $\mathcal{N}$ 上的全纯函数, 并且 $z_{j}(a) \neq z_{k}(a), k \neq j, k, j=0,1, \ldots, p(a)-1$. 否则, $z_{j}(a)$ 将是 $f_{a}^{p}(z)=z$ 的重根, 必有

$$
\left(f_{a}^{p}\right)^{\prime}\left(z_{j}(a)\right)=1
$$

与 $|\lambda(a)|<1$ 矛盾. 这意味着 $p=p(a)$ 在 $\mathcal{N}$ 上是常数, 从而乘子 $\lambda(a)$ 是 $\mathcal{N}$ 上全纯函数. 由于当 $a$ $\in \partial \mathcal{N}$ 时, $f_{a}$ 非双曲, 故当 $a \rightarrow \partial \mathcal{N}$ 时,

$$
|\lambda(a)| \rightarrow 1
$$

因此,

$$
\lambda: \mathcal{N} \rightarrow \mathbb{D}, \quad \lambda(a)=\left(f_{a}^{p}\right)^{\prime}\left(z_{0}(a)\right)
$$

是逆紧映射, 从而是全纯分支覆盖映射. 利用拟共形手术和 Ahlfors-Bers 可测 Riemann 映射定理, 可 得 $\lambda$ 是局部可逆的 (细节参见文献 [12]), 因而是一个全纯覆盖映射. 再由 $\mathbb{D}$ 是单连通的, 得到 $\lambda$ 是共 形映射. 故当 $a \in \mathcal{N} \rightarrow \alpha \in \partial \mathcal{N}$ 时, 有

$$
|\lambda(a)| \rightarrow 1 .
$$

由于周期点 $z_{0}(a)$ 事实上可以解析延拓到 $\partial \mathcal{N}$, 只要 $\lambda(a) \neq 1$. 而对应于 $\lambda(a)=1$ 的点是 $z_{0}(a)$ 的一个代数奇点. 从而, $\lambda(a)$ 也可解析延拓到 $\partial \mathcal{N}$, 除了 $\lambda(a)=1$ 时有代数奇点. 因此, $\partial \mathcal{N}$ 是由 $|\lambda(a)|=1$ 所确定的, 除了 $\lambda(a)=1$ 处可能例外的解析曲线. 而 $\lambda(a)=1$ 对应于 $\partial \mathcal{N}$ 上的唯一边界点 (由 Carathéodory 边界对应定理, 当 $z \rightarrow 1$ 时, $\lambda^{-1}(z) \rightarrow a \in \partial \mathcal{N}$ 极限存在且唯一). 因此, $\partial \mathcal{N}$ 是一条 解析曲线, 至多有一个对应于 $\lambda(a)=1$ 的例外点, 这是一个抛物参数, 也是唯一可能的尖点.

\section{参考文献}

1 Branner B, Hubbard J H. The iteration of cubic polynomials. Part I: The global topology of parameter space. Acta Math, 1988, 160: 143-206

2 Petersen C L, Tan L. Analytic coordinates recording cubic dynamics. In: Complex Dynamics: Families and Friends. Wellesley: A K Peters, 2009, 413-449

3 Milnor J. Cubic polynomial maps with periodic critical orbit: Part I. In: Complex Dynamics Families and Friends. Wellesley: A K Peters, 2009, 333-411

4 Faught D. Local connectivity in a family of cubic polynomials. PhD Thesis. Ithaca: Cornell University, 1992

5 Roesch P. Hyperbolic components of polynomials with a fixed critical point of maximal order. Ann Sci École Norm Sup (4), 2007, 40: 901-949

6 Carleson L, Jones P W, Yoccoz J C. Julia and John. Bol Soc Bras Mat, 1994, 25: 1-30

7 Roesch P, Yin Y. The boundary of bounded polynomial Fatou components. Comptes Rendus Math, 2008, 346: 877-880

8 Mañé R, Sad P, Sullivan D. On the dynamics of rational maps. Ann Sci École Norm Sup (4), 1983, 16: 193-217

9 Bers L, Royden H L. Holomorphic families of injections. Acta Math, 1986, 157: 259-286

10 Słodkowski Z. Holomorphic motions and polynomial hulls. Proc Amer Math Soc, 1991, 111: 347-355 
11 Ahlfors L, Bers L. Riemann's mapping theorem for variable metrics. Ann of Math (2), 1960, 72: 385-404

12 Carleson L, Gamelin T W. Complex Dynamics. New York: Springer, 1993

\section{Capture components are quasidisks: Cubic polynomials with a critical fixed point}

\section{Yueyang Wang \& Weiyuan Qiu}

Abstract According to the classification by Milnor, the hyperbolic components in the parameter plane of the cubic polynomials with a critical fixed point can be classified into three types: The principal hyperbolic component, the capture components, and the non-capture components. Roesch proved that cusps are dense on the boundary of the principal hyperbolic component. In this paper, we prove that the boundary of every capture component is a quasi-circle, which implies there are no cusps on it, while the boundary of a non-capture component is a smooth Jordan curve (except at most one cusp at the parabolic parameter).

Keywords cubic polynomial, hyperbolic component, capture component, quasi-circle, cusps, holomorphic motion

MSC(2010) 37F45, 37F10

doi: 10.1360/N012018-00235 